\title{
Methylated BNIP3 gene in colorectal cancer prognosis
}

\author{
SAYAKA SHIMIZU ${ }^{1}$, SATORU IIDA ${ }^{1}$, MEGUMI ISHIGURO $^{1}$, HIROYUKI UETAKE ${ }^{2}$, TOSHIAKI ISHIKAWA ${ }^{2}$, \\ YOKO TAKAGI ${ }^{2}$, HIROTOSHI KOBAYASHI ${ }^{1}$, TETSURO HIGUCHI ${ }^{1}$, MASAYUKI ENOMOTO $^{1}$, \\ KAORU MOGUSHI ${ }^{3}$, HIROSHI MIZUSHIMA ${ }^{3}$, HIROSHI TANAKA ${ }^{3}$ and KENICHI SUGIHARA ${ }^{1}$
}

\author{
Departments of ${ }^{1}$ Surgical Oncology, ${ }^{2}$ Translation Oncology and the ${ }^{3}$ Information Center for Medical Sciences, \\ Graduate School, Tokyo Medical and Dental University, Tokyo 113-8519, Japan
}

Received June 7, 2010; Accepted July 19, 2010

DOI: 10.3892/ol_00000153

\begin{abstract}
The DNA methylation of apoptosis-related genes in various cancers contributes to the disruption of the apoptotic pathway and results in resistance to chemotherapeutic agents. Irinotecan (CPT-11) is one of the key chemotherapy drugs used to treat metastatic colorectal cancer (CRC). However, a number of metastatic CRC patients do not benefit from this drug. Thus, the identification of molecular genetic parameters associated with the response to CPT-11 is of interest. To identify apoptosis-related genes that may contribute to CPT-11 resistance, microarray analysis was conducted using colon cancer cells in which 5-aza-2'deoxycytidine (DAC) enhanced sensitivity to CPT-11. Microarray analysis identified 10 apoptosis-related genes that were up-regulated following treatment with DAC. Among the genes, Bcl-2/adenovirus E1B $19 \mathrm{kDa}$ protein interacting protein 3 (BNIP3), a Bcl-2 family pro-apoptotic protein, was identified as being involved in CPT-11 resistance following methylation of its promoter. An analysis of 112 primary CRC cases revealed that approximately $58 \%$ of cases showed BNIP3 methylation, and that patients with methylation exhibited a poorer outcome compared to those without methylation. In addition, in 30 patients who received first-line CPT-11 chemotherapy, patients with methylation exhibited resistance to chemotherapy compared to patients with no methylation. The results suggest that methylation of BNIP3 is a predictive factor in the prognosis and response to CPT-11 treatment in CRC patients.
\end{abstract}

\section{Introduction}

Colorectal cancer (CRC) is the third most common cancer worldwide, and the second most common cause of cancer-related death (1). Approximately $20-40 \%$ of CRC patients that undergo curative surgery develop local recurrence or distant metastasis,

Correspondence to: Dr S Iida, Department of Surgical Oncology, Tokyo Medical and Dental University, 1-5-45, Yushima, Bunkyo-ku, 1138519 Tokyo, Japan

E-mail: s-iida.srg2@tmd.ac.jp

Key words: methylation, colorectal cancer, prognosis, apoptosis, irinotecan and exhibit a poor outcome that is generally less than 2 years (2). Chemotherapy is an important strategy in the treatment of metastatic CRC, and irinotecan (CPT-11) is one of the major chemotherapy drugs used in metastatic CRC treatment. Treatment with a combination of CPT-11, 5-fluorouracil (5-FU) and leucovorin is generally approved as the standard chemotherapy for metastatic disease and somewhat increases survival (3). However, the majority of patients eventually succumb to the disease. Various predictive factors of chemosensitivity were previously investigated (4). Regarding 5-FU chemotherapy, the target enzyme thymidylate synthase and the metabolic enzyme dihydropyrimidine dehydrogenase were suggested as predictive factors (4). The predictive factors underlying CPT-11 chemosensitivity have yet to be sufficiently investigated. Thus, identification of the molecular genetic parameters associated with response to CPT-11 is of clinical interest.

Apoptosis or programmed cell death occurs in various physiological and pathological situations (5). It occurs not only in normal tissues but also in malignant tumors, and plays an important role in both the pathogenesis of tumors and their biological behavior (6). Defects in the apoptotic pathway contribute to cell accumulation in the colon, promoting malignancy and subsequent metastasis, and allow tumor cells to survive in a suspended state, thereby permitting their hematogenous or lymphatic dissemination (7). The apoptotic pathway may also be a final common step in the cytotoxicity exerted by a number of anticancer drugs with various underlying mechanisms of action $(8,9)$. The altered expression of genes that encode apoptotic proteins provide cells with inherent resistance to anticancer drugs. Thus, the expression levels of apoptotic proteins serves as a predictor of chemotherapeutic agent response.

In addition to genetic aberrations such as mutation and deletion, increasing evidence suggests that epigenetic changes play an important role in CRC pathogenesis $(10,11)$. The aberrant methylation of gene promoter regions leading to gene silencing is currently the most widely studied epigenetic abnormality in human malignancies, affecting multiple cellular functions including cell growth and differentiation, cell cycle control and DNA repair $(11,12)$. Given that DNA methylation affects the transcription of crucial genes involved in the regulation of apoptosis $(13,14)$, it may also contribute to the evolution of resistance to chemotherapeutic agents. 
In a previous study, it was shown that the inactivation of apoptosis-related genes due to DNA hypermethylation may predict the response to the 5-FU-based chemotherapy treatment of gastric cancer (15). To identify the apoptosis-related genes involved in reduced sensitivity to CPT-11, the genome was screened for genes responding to the demethylating agent 5-aza-2'-deoxycytidine (DAC) using microarray analysis. Colon cancer cells were used in the event that DAC was able to enhance sensitivity to CPT-11. Based on our microarray results, an apoptosis-related gene, Bcl-2/adenovirus E1B $19 \mathrm{kDa}$ protein interacting protein 3 (BNIP3)was selected. The methylation status of the gene was analyzed in primary CRCs. Moreover, the methylation status was compared with various clinicopathological variables and BNIP3 methylation was investigated as a predictor of prognosis and response to CPT-11 treatment in CRC patients.

\section{Materials and methods}

Reagents. SN-38, an active metabolite of CPT-11, was provided by Yakult (Tokyo, Japan). SN-38 was dissolved in dimethyl sulfoxide at a concentration of $1 \mu \mathrm{M}$ and stored at $-20^{\circ} \mathrm{C}$, before further dilution in phosphate-buffered saline (PBS) and filter sterilization immediately prior to use. DAC was obtained from Sigma (St. Louis, MO, USA), dissolved in PBS and filter-sterilized.

Cell lines. The human colon adenocarcinoma cell lines HCT-15 and HT29 were provided by the Cell Resource Center for Biomedical Research, Tohoku University (Miyagi, Japan) and the American Type Culture Collection (Manassas, VA, USA). HCT-15 cells were maintained in RPMI-1640 (Sigma) containing $10 \%$ heat-inactivated fetal bovine serum (FBS), $100 \mathrm{U} / \mathrm{ml}$ penicillin, $100 \mu \mathrm{g} / \mathrm{ml}$ streptomycin, $10 \mathrm{mM}$ HEPES (Gibco-BRL, Gaithersburg, MD, USA) and $1.0 \mathrm{mM}$ sodium pyruvate (Gibco). HT29 cells were maintained in Mac Coy's medium containing $10 \%$ heat-inactivated FBS, $100 \mathrm{U} / \mathrm{ml}$ penicillin and $100 \mu \mathrm{g} / \mathrm{ml}$ streptomycin. The cell lines were cultured at $37^{\circ} \mathrm{C}$ in $5 \% \mathrm{CO}_{2}$. Cells $\left(5 \times 10^{3}\right.$ per well) were plated in 24-well plates on day 1 of the culture. On day 3, the medium was removed and new medium containing DAC was added. The cells were treated with $0.5 \mu \mathrm{M}$ DAC (or PBS as a control) for $72 \mathrm{~h}$. On day 6 , the cells were rinsed twice with FBS-free medium, and fresh medium containing $0.0015 \mu \mathrm{M} \mathrm{SN}-38$ (or PBS) was added. The dose of each drug was administered on the basis of its pharmacological dose as previously reported (14), and our preliminary experiments (data not shown). The cells were harvested following incubation with trypsin-EDTA on day 9 , stained with trypan blue and counted. Total mRNA or genomic DNA was extracted on day 6 and utilized in the analysis of mRNA expression or methylation status following DAC treatment.

Microarray analysis. Total RNA was extracted from cultured cells using the RNeasy kit (Qiagen, Hilden, Germany). The integrity of the RNA was assessed using the Agilent 2100 BioAnalyzer (Agilent Technologies, Palo Alto, CA, USA), and the samples were confirmed to have an RNA Integrity Number $>5.0$ prior to gene expression analysis. Contaminant DNA was removed via digestion with RNase-free DNase (Qiagen).
Using $2 \mu \mathrm{g}$ total RNA, cRNA was prepared using the onecycle target labeling and control reagents kit (Affymetrix, Santa Clara, CA, USA). Hybridization and signal detection of HG-U133 plus 2.0 arrays (Affymetrix) were performed following the manufacturer's instructions. The 4 microarray datasets obtained were normalized using the robust multi-array average (RMA) method (16) and R 2.4.1 statistical software (17) together with a BioConductor package. Normalized gene expression levels were log2-transformed, and 62 control probe sets were removed for further analysis. For each of the 54,613 probe sets, a fold change analysis was performed using each pair of treated and untreated HCT15 and HT29 cells. Probe sets that showed $>1.5$-fold increase or decrease were identified as differentially expressed genes.

Real-time polymerase chain reaction (RT-PCR). Total cellular RNA was extracted from the treated cells using the RNeasy Mini Kit (Qiagen, Valencia, CA, USA) and incubated with DNase as previously reported (14). cDNA was then synthesized from $10 \mathrm{ng}$ extracted RNA using the High-Capacity cDNA reverse transcription kit (Applied Biosystems). PCR was performed as previously described (14), using the conditions: initial denaturation for $96^{\circ} \mathrm{C}$ for $2 \mathrm{~min}$, followed by 26 cycles of denaturation for $1 \mathrm{~min}$ at $96^{\circ} \mathrm{C}$, annealing for $1 \mathrm{~min}$ at $57-60^{\circ} \mathrm{C}$ and extension for $1 \mathrm{~min}$ at $72^{\circ} \mathrm{C}$. As an internal control for RT-PCR analysis, glyceraldehyde-3-phosphate dehydrogenase (GAPDH) transcripts were amplified from the same cDNA samples. Primer sequences were: GAPDH, 5'-CAACAGCCTCAAGATCATCAGC-3' (forward) and 5'-TCCTAGACGGCAGGTCAGGTC-3' (reverse); BNIP3, 5'-GGATGCAGGAGGAGAGCCT-3' (forward) and 5'-CGAG GTGGGCTGTCACAGT-3' (reverse); and SOCS3, 5'-GACCA GCGCCACTTCTT-CAC-3' (forward) and 5'-ACTGGATGCG CAGGTTCTTG-3' (reverse). Following amplification, PCR products were separated on $2 \%$ agarose gels, stained with ethidium bromide and visualized under ultraviolet illumination.

DNA extraction. Tumor or normal mucosal tissue was cut into $10-\mu \mathrm{m}$ sections from tissue blocks, with a change of blade and cleaning of the microtome between specimens. The specimens were deparaffinized and washed, and tumor tissue was manually dissected using a razor blade, using a hematoxylin and eosin (H\&E)-stained slide as a guide. Dissected tissues or cultured cell lines were then incubated overnight with proteinase-K in digestion buffer and the genomic DNA was extracted using a standard phenol chloroform method.

Methylight analysis. Sodium bisulfite conversion and DNA recovery were performed using EpiTect Bisulfite (Qiagen). Following sodium bisulfite conversion, the genomic DNA was analyzed using the Methylight technique, a fluorescencebased RT-PCR (Q-PCR) assay (18) and the ABI Prism 7300 R-T PCR System (Taq Man; Applied Biosystems, Foster City, CA, USA). Two sets of primers and probes, designed specifically for bisulfite-converted DNA were used. One set detected methylation in the gene of interest and the second set served as a reference set for $\beta$-actin (ACTB) to normalize for input DNA. The reference primers and probes were designed in a region of the ACTB gene that lacks $\mathrm{CpG}$ dinucleotides, allowing for equal amplification regardless of the methylation levels. Primer 
and probe sequences were: ACTB, 5'-AGGTTGGGGAAGTTT GTTTTTG-3' (forward), 5'-CCACCACCCAACACACAATA-3' (reverse) and 5'-TGGGGTGGTGATGGAGGAGGTT-3' (probe); BNIP3, 5'-TTCGGTCGGAGGAATTTATAGG-3' (forward), 5'-CCCCAATCGCGACCAA-3' (reverse) and 5'-ACGACGCGACCGCAAAT-3' (probe); as well as SOCS3 5'-TCGCGTTTTTTT-TTTCGTAGTTT-3' (forward), 5'-CGC GACCTCCGCACA-3' (reverse) and 5'-CGACCGCTACCG CATCCCGA-3' (probe). SssI-treated HCT-15 DNA was used as a fully methylated positive control (100\% methylation ratio). Parallel Taq Man PCR reactions were performed with specific primers for the bisulfite-converted methylated sequence for a particular locus and with the ACTB reference primers. In each case, triplicate threshold cycle $(\mathrm{Ct})$ values were obtained and averaged, and expression levels were then evaluated by the $2-\Delta \Delta \mathrm{Ct}$ method (19). As an internal standard, each individual sample was normalized to its ACTB content and compared to the gene expression level of SssI-treated HCT-15 DNA as positive controls (calibration sample) as follows: $2^{\Delta \Delta C t}$, where $\Delta \Delta \mathrm{Ct}=(\mathrm{Ct}$-target-Ct-reference $)$-treated sample $-(\mathrm{Ct}$-target-Ctreference) calibrator sample. The percentage of fully-methylated reference (PMR) was defined as $2^{\Delta \Delta \mathrm{Ct}} \times 100 \%$.

Patients and tissue samples. Patients entered into this study had undergone surgical resection for primary sporadic colorectal cancer at the Department of Surgical Oncology, Tokyo Medical and Dental University (Tokyo, Japan). This study was pre-approved by the institutional review board of Tokyo Medical and Dental University, and written informed consent was obtained from all 112 participants prior to the commencement of the study. Following surgery for both colon and rectal cancer, patients exhibiting stage III cancer received oral or intravenous 5-FU-based adjuvant chemotherapy, and those with stage IV tumor or following recurrence received 5-FU-based systemic chemotherapy. The resected specimens were fixed in $10 \%$ neutral buffered formalin and embedded in paraffin. For all cases, archival H\&E slides of the primary tumors were retrieved and reviewed to confirm pathological features. In addition to the International Union Against Cancer Tumor-Node-Metastasis (TNM) classification (20), clinicopathological factors such as age, degree of histological differentiation, pathological tumor class and lymph node metastasis at the time of diagnosis in stage I-III patients during the follow-up period were studied. The response rate to chemotherapy including CPT-11 treatment was assessed by Response Evaluation Criteria In Solid Tumors (21).

Statistical analysis. Statistical analysis was carried out using StatView Software (version 5.0). To estimate the differences between groups, the Chi-square test, Welch's t-test and log-rank test were used where appropriate. The Kaplan-Meier method was used to estimate survival. Survival was calculated from the date of surgery. Prognostic factors were examined by univariate and multivariate analysis using the Cox proportional hazards model. $\mathrm{P}<0.05$ was considered to be statistically significant.

\section{Results}

DAC increased sensitivity to $S N-38$ in colorectal cancer cells. Pre-incubation with the demethylating agent DAC was examined to investigate whether it influenced the sensitivity of the colon cancer cell lines HCT-15 and HT29 to SN-38. On day 9, the total number of HCT-15 cells that had been pre-incubated with DAC prior to exposure to $\mathrm{SN}-38$ was found to be significantly decreased in comparison to cells treated with SN-38 alone ( $p=0.0049$, Fig. 1A). A similar reduction was observed in the HT29 cells $(\mathrm{p}=0.0038$, Fig. 1A). Treatment with DAC or SN-38 alone resulted in no significant effect on the rate of cell proliferation in the two cell lines.

\section{Identification of pro-apoptotic genes induced by DAC in colon} cancer cells. Since inactivation of the apoptotic pathway is associated with chemoresistance (8), identification of the proapoptotic genes that may be reactivated by DAC was attempted. Using oligonucleotide microarrays, the global changes in gene expression following the treatment of HCT-15 and HT29 with DAC were analyzed, and the expression changes in treated and untreated cells were compared. Expression levels of 943 genes were found to be increased by $>1.5$-fold following DAC treatment in HCT-15 cells, and 2,720 genes were increased in the HT29 cells. A total of 234 of these genes were found to be similarly altered in the two cell lines. Among the 234 genes, 10 were identified as having known pro-apoptotic activities (Table I). As the expression levels of BNIP3 and SOCS3 are frequently reduced due to promoter methylation in gastrointestinal cancers $(22,23)$, up-regulation of these genes following DAC treatment was analyzed to determine whether it was caused by DNA demethylation. The BNIP3 mRNA expression was up-regulated and its DNA promoter was demethylated in both the HCT-15 and HT29 cell lines following treatment with DAC (Fig. 1B and C). In contrast, although SOCS3 mRNA expression was enhanced after DAC treatment, primers and probes specific for methylated SOCS3 failed to amplify any product in the two cell lines by Methylight analysis (data not shown). The results indicated that SOCS3 expression may be induced by DAC treatment independently of the methylation status of the DNA promoter.

Relationship between clinicopathological factors and BNIP3 methylation in CRC patients. The median PMR value for BNIP3 methylation in 16 randomly selected patients with normal colon epithelial tissue was 0 . The positive methylation was defined as PMR $>0$. The BNIP3 methylation status was then investigated in 112 patients who had undergone surgical resection for primary CRC between March 2000 and April 2003. Patients were prospectively followed over a median post-operative duration of 42 months and methylation of $B N I P 3$ was detected in $58 \%$ of the 112 patients. No significant correlations were found between BNIP3 methylation and clinicopathological factors such as age, gender, tumor depth, vessel invasion, lymphatic invasion, lymph node metastasis and stage (Table II). Patients with BNIP3 methylation also exhibited a significantly shorter overall survival time (OS) compared to those without methylation ( $\mathrm{p}=0.012$, Fig. $2 \mathrm{~A}$ ). In stage II/III tumors, patients with BNIP3 methylation also exhibited a significantly shorter OS than those without methylation ( $p=0.039$, Fig $2 \mathrm{~B}$ ). No difference in relapse-free survival (RFS) was observed between patients with or without methylation ( $\mathrm{p}=0.16$, Fig. $2 \mathrm{C}$ ). Regarding stage II/III patients, investigation of the univariate analysis of survival using the 
A
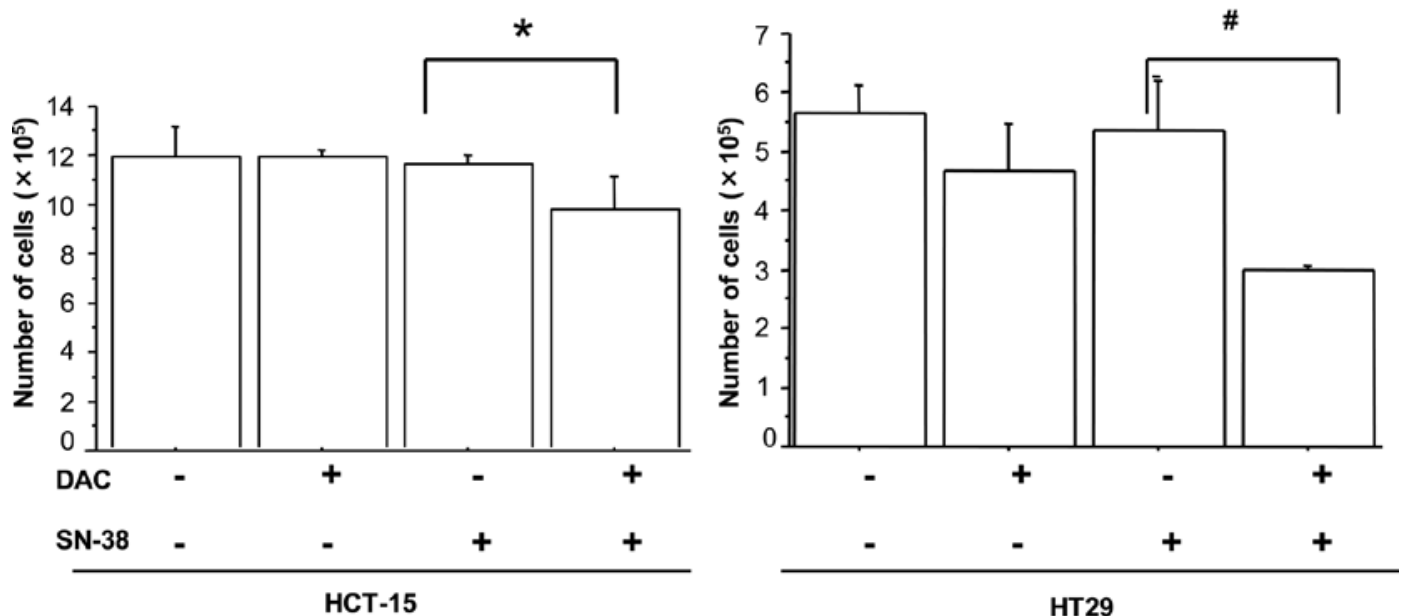

B

C
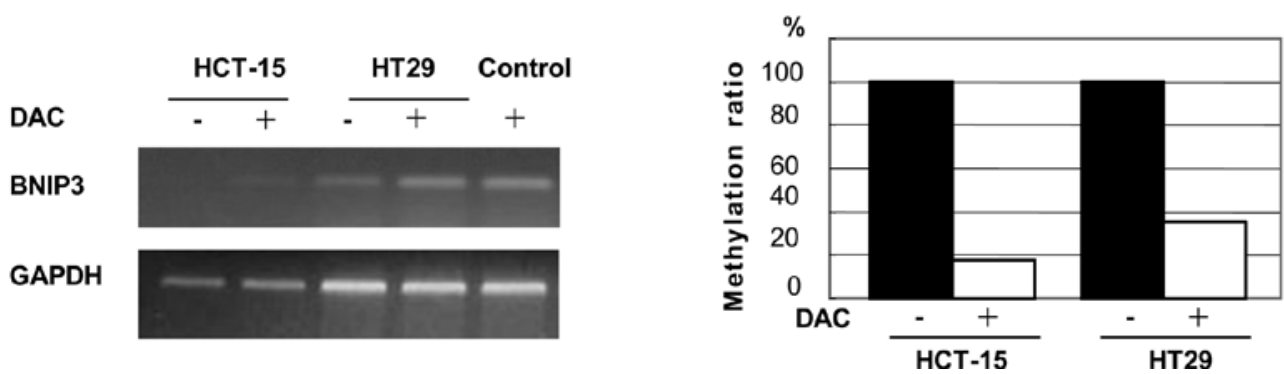

Figure 1. (A) In vitro growth inhibition following treatment with the demethylating agent DAC and/or SN-38. HCT-15 and HT29 cells were treated with 4 protocols (untreated, DAC alone, SN-38 alone or DAC followed by SN-38), and the total number of cells were counted on day 9. (B) Changes in mRNA expression and $(\mathrm{C})$ the methylation status of BNIP3 as examined by RT-PCR and Methylight analysis after DAC treatment, respectively. Error bars are the mean \pm SD. ${ }^{*} \mathrm{p}=0.0049,{ }^{*} \mathrm{p}=0.0038$.

Table I. Apoptosis-related genes up-regulated by DAC treatment in colon cancer cell lines.

Fold change

\begin{tabular}{llrr} 
Symbol & Name & HCT-15 & HT29 \\
\hline UNC5B & Unc-5 homolog B (C. elegans) & 5.21 & 11.8 \\
NUPR1 & Nuclear protein 1 & 2.26 & 5.68 \\
ATF5 & Activating transcription factor 5 & 4.76 & 3.00 \\
IFI6 & Interferon, $\alpha$-inducible protein 6 & 2.92 & 3.28 \\
MX1 & Myxovirus (influenza virus) resistance 1, interferon-inducible protein p78 (mouse) & 2.07 & 3.34 \\
SCIN & Scinderin & 6.55 & 2.34 \\
STAT1 & Signal transducer and activator of transcription 1, 91 kDa & 2.03 & 1.80 \\
SOCS3 & Suppressor of cytokine signaling 3 & 3.48 & 1.61 \\
BNIP3 & BCL2/adenovirus E 1B 19 kDa interacting protein 3 & 22.1 & 1.54 \\
EEF1A2 & Eukaryotic translation elongation factor $\alpha 2$ & 1.51 & 1.67 \\
\hline
\end{tabular}

Cox proportional hazards model showed that BNIP3 methylation $(\mathrm{p}=0.043)$ and lymph node metastasis $(\mathrm{p}=0.0086)$ were significantly associated with OS. All of the variables with a significance level at $\mathrm{p}<0.35$ in the univariate analyses were subsequently used for multivariate analyses. Multivariate analysis of the variables failed to show BNIP3 methylation to be an independent prognostic factor (Table III).

Prognostic value of BNIP3 methylation in CRC patients who received treatment with chemotherapeutic agents containing 
Table II. Relationship between clinicopathological factors and BNIP3 methylation.

\begin{tabular}{|c|c|c|c|}
\hline & \multicolumn{3}{|c|}{$B N I P 3$} \\
\hline & Met $(n=65)$ & $\operatorname{Unm}(\mathrm{n}=47)$ & p-value \\
\hline Age & & & 0.88 \\
\hline$<64$ & 30 & 21 & \\
\hline$\geq 64$ & 35 & 26 & \\
\hline Gender & & & 0.48 \\
\hline Male & 43 & 28 & \\
\hline Female & 22 & 19 & \\
\hline Location & & & 0.88 \\
\hline Right & 24 & 18 & \\
\hline Left & 41 & 29 & \\
\hline Lymphatic invasion & & & 0.25 \\
\hline Negative & 12 & 13 & \\
\hline Positive & 53 & 34 & \\
\hline Vessel invasion & & & 0.88 \\
\hline Negative & 5 & 4 & \\
\hline Positive & 60 & 43 & \\
\hline Depth of invasion & & & 0.23 \\
\hline $\mathrm{T} 1, \mathrm{~T} 2$ & 14 & 6 & \\
\hline $\mathrm{T} 3, \mathrm{~T} 4$ & 51 & 41 & \\
\hline Lymph node metastasis & & & 0.24 \\
\hline Negative & 30 & 27 & \\
\hline Positive & 35 & 20 & \\
\hline Stage & & & 0.19 \\
\hline I & 9 & 4 & \\
\hline II & 17 & 21 & \\
\hline III & 25 & 16 & \\
\hline IV & 14 & 6 & \\
\hline
\end{tabular}

Met, methylation group; Unm, unmethylation group.

CPT-11. BNIP3 methylation was examined to determine whether it was a prognostic marker in primary CRC patients treated with CPT-11. Of the patients who had undergone surgical resection for primary CRC between May 1998 and September 2007, 30 patients (including 5 from 112 patients between 2000 and 2003) who demonstrated recurrence after radical resection or incomplete resection due to distant metastasis, received first-line chemotherapy with CPT-11. Patients were subsequently observed over a median duration of 15 months following the initial administration of CPT-11. One patient had complete response (CR), 10 partial response (PR), 7 stable disease (SD) for $>6$ months, and 12 progression of disease (PD). When CR and PR patients were defined as responders, the observed response rate was lower in patients with BNIP3 methylation than those without (33.3 vs. $40 \%$ ), but this difference was not statistically significant $(\mathrm{p}=0.70$, Fig. 3A). Time to progression (TTP) in patients with BNIP3
A

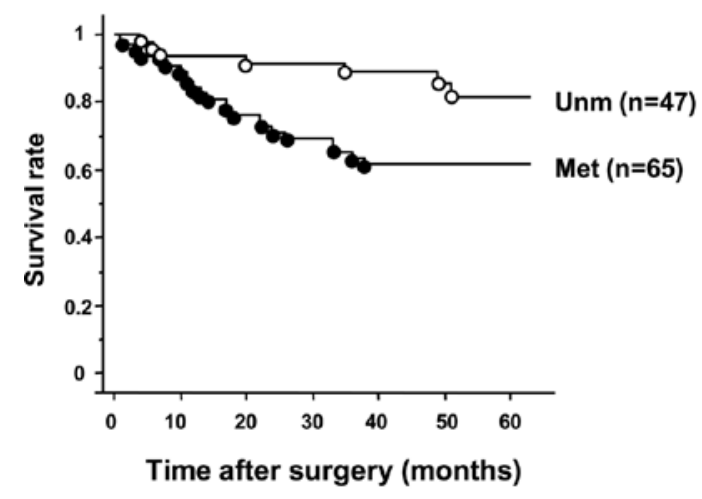

B

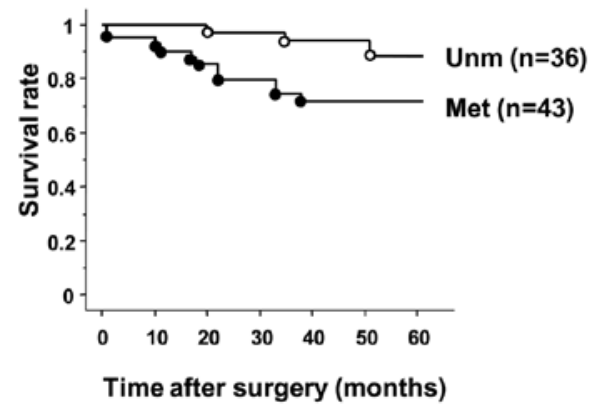

C

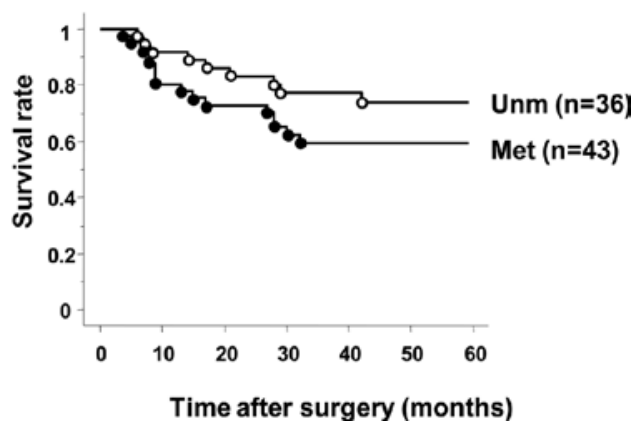

Figure 2. Survival analysis according to the presence of $B N I P 3$ methylation. (A) OS of the 112 patients. Patients with BNIP3 methylation exhibited a significantly shorter OS compared to those without methylation $(\mathrm{p}=0.012)$. (B) OS of stage II/III patients. OS of patients with methylation was significantly shorter compared with that of patients without methylation $(\mathrm{p}=0.039)$. (C) RFS of stage II/III patients. RFS of patients with methylation was shorter than that of patients without methylation, but this difference did not reach statistical significance $(\mathrm{p}=0.16)$. $\bullet$, methylation group $(\mathrm{Met}) ; \circ$, unmethylation group (Unm)

methylation was slightly shorter than that in patients without methylation, but this difference was not statistically significant (median 196.5 vs. 237 days) (p=0.10, Fig. 3B).

\section{Discussion}

In this study, 2 colon cancer cell lines were utilized in which DAC treatment enhanced sensitivity to $\mathrm{SN}-38$, and identified the apoptosis-related genes that were up-regulated following treatment with DAC by microarray analysis. Among these genes, we confirmed methylation of the BNIP3 promoter 
Table III. Analysis of overall survival in stage II/III patients using the Cox proportional hazard model.

Stage II/III patients $(\mathrm{n}=79)$

\begin{tabular}{llll} 
Prognosis factor & Hazard ratio & $95 \%$ CI & p-value \\
\hline Univariate analysis & & & 0.043 \\
$B N I P 3$ methylation & 3.744 & $1.044-13.432$ & 0.34 \\
Age & 1.647 & $0.579-4.819$ & 0.17 \\
Gender & 2.457 & $0.684-8.850$ & 0.37 \\
Tumor differentiation & 0.555 & $0.155-1.993$ & 0.36 \\
Tumor location & 0.614 & $0.215-1.752$ & 0.88 \\
Depth of invasion & 1.170 & $0.153-8.929$ & 0.0086 \\
Lymph node metastasis & 4.739 & $1.484-15.152$ & 0.33 \\
Lymphatic invasion & 2.762 & $0.361-21.277$ & 0.075 \\
Multivariate analysis & & & 0.57 \\
$B N I P 3$ methylation & 3.260 & $0.887-11.984$ & 0.26 \\
Age & 1.366 & $0.468-3.985$ & 0.15 \\
Gender & 2.105 & $0.574-7.752$ & 0.75 \\
Lymph node metastasis & 3.040 & $0.670-13.889$ & $0.130-16.928$ \\
Lymphatic invasion & 1.481 & & \\
\hline
\end{tabular}

A

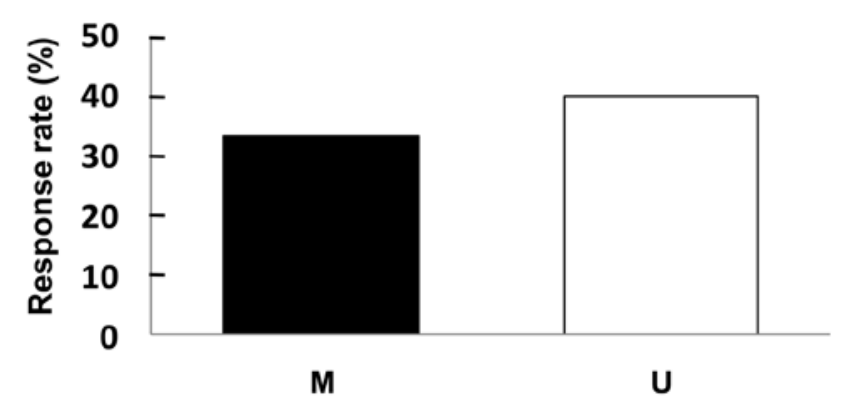

B

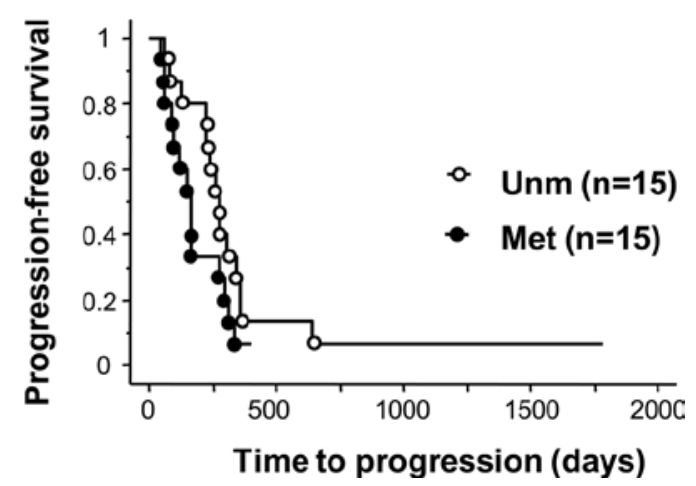

Figure 3. Relationship between the response to CPT-11 and BNIP3 methylation in 30 patients that received first-line CPT-11 chemotherapy. (A) Response rate to CPT-11. Response rate of patients with BNIP3 methylation (M) was lower than that of patients without (U) (33.3 vs. $40 \%$ ), although differences did not reach statistical significance $(\mathrm{p}=0.70)$. (B) Time to progression $(\mathrm{TTP})$ among patients who received CPT-11. TTP of patients with methylation was shorter than that of patients without methylation (196.5 vs. 237 days), but this difference was not statistically significant (p=0.10). $\bullet$, methylation group (Met); o, unmethylation group (Unm).

in colon cancer cell lines, and subsequently detected its methylation in more than half of the cases of primary CRC. Our results showed that BNIP3 methylation is associated with poor clinical outcome and chemoresistance.

Hypoxia is a common phenomenon observed in solid tumors and is known to stabilize hypoxia-inducible factor-1 (HIF-1), which is involved in the pathways underlying angiogenesis, glucose metabolism and cell proliferation (24-26). HIF-1 transactivates a large number of genes that may either promote or inhibit the growth and survival of individual tumor cells (27). Thus, selection of an individual tumor cell may maintain the expression of beneficial HIF-1 transcriptional targets, while silencing pro-death hypoxia-induced signals may result in lethal malignancy (28). BNIP3 is a pro-apoptotic member of the Bcl-2 family whose role is mediated by HIF-1 (29-31). $B N I P 3$ is also known to play an important role in the regulation of apoptosis (32-34). An increased BNIP3 expression induces cell death through mitochondrial dysfunction, membrane depolarization, mitochondrial permeability transition pore opening and increased production of reactive oxygen species (35). A reduced $B N I P 3$ expression has been identified in a wide range of cancer cells and primary malignancies $(23,36,37)$. A number of recent studies showed that methylation of the BNIP3 promoter may play an important role in silencing expression in a range of tumor types. These studies also showed that BNIP3 methylation is detected in various primary cancers $(23,36,37)$. In this study, BNIP3 methylation was present in almost $60 \%$ of the primary CRC cases. Taken together, silencing BNIP3 by 
methylation may be important in tumorigenesis processes in the colon and rectum. Given the potential ability of HIF-1 to evoke apoptosis through its target gene, the down-regulation of BNIP3 by methylation may disrupt the HIF-1-BINP3 apoptotic pathway and permit cancer cells with high malignant potential to survive, since CRC patients with BNIP3 methylation had a poorer outcome than those with without. It was reported that BNIP3 silencing induces metastatic growth of breast cancer in the liver, lung and bone (28). In this study, $70 \%(14 / 20)$ of patients with distant metastasis exhibited BNIP3 methylation in the $112 \mathrm{CRC}$ cases examined (Table II), suggesting that BHIP3 silencing may contribute to the acquisition of metastatic potential in cancer cells.

CPT-11 is a topoisomerase I (topo-I) inhibitor that forms stable topo-I DNA-cleavable complexes and inhibits the progression of the replication fork. However, the relationship between the expression levels of topo-I mRNA and chemosensitivity of CPT-11 is unclear $(38,39)$. In a previous study, we showed that DAC increases the growth inhibitory effects of CPT-11 on the colon cancer cell line HCT-15, despite showing no effect on topo-I expression levels (14). Furthermore, our microarray experiment did not identify changes in topo-I expression following treatment with DAC. Thus, topo-I may not be involved in the enhanced sensitivity to CPT-11 following DAC treatment.

Apoptosis is a significant mechanism through which chemotherapeutic agents exert their cytotoxic effects on cancer cells. However, cancer cells acquire resistance to apoptosis due to an altered expression or mutation of apoptosis-related genes during the tumorigenesis processes (40). Numerous studies have shown a relationship between disruption of the apoptosis pathway and chemoresistance in various tumors $(8,9)$. In addition, the susceptibility of cancer cells to cytotoxic drugs appears to be at least partially due to a dependence on the balance between pro- and anti-apoptotic members of the Bcl-2 family (41). Given that BNIP3 is a member of the Bcl-2 family of pro-apoptotic proteins, and that it appears to antagonize the activity of pro-survival proteins, including Bcl-2 and Bcl-xL (35), it may also contribute to chemosensitivity. Previously, BNIP3 expression was found to be down-regulated in clones with acquired resistance to 5-FU compared to their parental CRC cell lines (33). Additionally, BNIP3 expression was found to be associated with paclitaxel response in an ovarian cancer model (42). BNIP3 down-regulation results from the addition of small interfering RNA enhanced chemoresistance in pancreatic cancer cells $(43,44)$. By contrast, the overexpression of $B N I P 3$ in rat fibroblast cells increased sensitivity to apoptosis induced by topo-I and -II inhibitors (45). Our previous study showed that the inhibitory effects of SN-38 on tumor tissue are increased in a CRC xenograft model when BNIP 3 expression is restored via promoter demethylation following treatment with DAC (14). In this study, BNIP3 was up-regulated following treatment with DAC in SN-38-resistant CRC cells when DAC increased sensitivity to SN-38. Moreover, CRC patients with BNIP3 methylation exhibited a shorter TTP for CPT-11 treatment. Thus, BNIP3 may play an important role in the reduced response to CPT-11 treatment in CRC patients.

In conclusion, the relatively high frequency of BNIP3 methylation in primary CRC suggests that this gene is involved in carcinogenesis of the colon and rectum. Moreover, since
BNIP3 methylation is associated with poor OS and decreased sensitivity to CPT-11, the methylation status of this gene may be a predictive factor for prognosis and responsiveness to CPT-11 in CRC patients. Since methylation appears to be reversed by a chemical agent, $B N I P 3$ reactivation via a demethylating agent may be a novel target for the treatment of CRC.

\section{References}

1. Ricchi P, Zarrilli R, Di Palma A and Acquaviva AM: Nonsteroidal anti-inflammatory drugs in CRC: from prevention to therapy. Br J Cancer 88: 803-807, 2003.

2. Kobayashi H, Mochizuki H, Sugihara K, et al: Characteristics of recurrence and surveillance tools after curative resection for colorectal cancer, a multicenter study. Surgery 141: 67-75, 2007.

3. Tournigand C, André T, Achille E, Lledo G, Flesh M, MeryMignard D, Quinaux E, Couteau C, Buyse M, Ganem G, Landi B, Colin P, Louvet C and de Gramont A: FOLFFIRI followed by FOLFOX6 or the reverse sequence in advanced colorectal cancer: a randomized GERCOR study. J Clin Oncol 22: 229-237, 2004

4. Ichikawa W, Uetake H, Shirota Y, Yamada H, Nishi N, Nihei Z, Sugihara K and Hirayama R: Combination of dihydropyrimidine dehydrogenase and thymidilate synthase gene expressions in primary tumors as predictive parameters for the efficacy of fluoropyrimidine-based chemotherapy for metastatic colorectal cancer. Clin Cancer Res 9: 86-91, 2003.

5. Hengarter MO: The biochemistry of apoptosis. Nature 407: 770-776, 2000.

6. Wyllie AH: Apoptosis. Br J Cancer 67: 205-208, 1993.

7. Compagni A and Chiristofori G: Recent advances in research on multistage tumorigenesis. Br J Cancer 83: 1-5, 2000.

8. Makin G and Dive C: Apoptosis and cancer chemotherapy. Trends Cell Biol 11: S22-S26, 2001.

9. Fulda S and Debatin KM: Extrinsic vs. intrinsic apoptosis pathways in anticancer chemotherapy. Oncogene 25: 4798-4811, 2006.

10. Suzuki H, Gabrielson E, Chen W, Anbazhagan R, van Engeland M, Weijenberg MP, Herman JG and Baylin SB: A genomic screen for genes upregulated by demethylation and histone deacetylase inhibition in human colorectal cancer. Nat Genet 31: 141-149, 2002.

11. Kondo Y and Issa JP: Epigenetic changes in colorectal cancer. Cancer Metastasis Rev 23: 29-39, 2004.

12. Jones PA and Baylin SB: The fundamental role of epigenetic events in cancer. Nat Rev Genet 3: 415-428, 2002.

13. Teodoridis JM, Strathdee G and Brown R: Epigenetic silencing mediated by $\mathrm{CpG}$ island methylation: potential as a therapeutic target and as a biomarker. Drug Resist Updat 7: 267-278, 2004.

14. Ishiguro M, Iida S, Uetake H, Morita S, Makino H, Kato K, Takagi Y, Enomoto M and Sugihara K: Effect of combined therapy with low-dose 5-aza-2'-deoxycytidine and irinotecan on colon cancer cell line HCT-15. Ann Surg Oncol 14: 1752-1762, 2007.

15. Kato K, Iida S, Uetake H, Takagi Y, Yamashita T, Inokuchi M, Yamada H, Kojima K and Sugihara K: Methylated TMS1 and DAPK genes predict prognosis and response to chemotherapy in gastric cancer. Int J Cancer 122: 603-608, 2008.

16. R, Development Core Team: R, A language and environment for statistical computing; R, Foundation for Statistical Computing, Vienna, Austria. ISBN 3-900051-07-0, URL http://www.Rproject.org, 2006.

17. Irizarry RA, Hobbs B, Collin F, Beazer-Barclay YD, Antonellis KJ, Scherf U and Speed TP: Exploration, normalization, and summaries of high density oligonucleotide array probe level data. Biostatistics 4: 249-264, 2003.

18. Eads CA, Danenberg KD, Kawakami K, Saltz LB, Blake C, Shibata D, Danenberg PV and Laird PW: MethyLight: a highthroughput assay to measure DNA methylation. Nucleic Acids Res 28: E32, 2000.

19. Shirota Y, Stoehlmacher J, Brabender J, Xiong YP, Uetake H, Danenberg KD, Groshen S, Tsao-Wei DD, Danenberg PV and Lenz HJ: ERCC1 and thymidylate synthase mRNA levels predict survival for colorectal cancer patients receiving combination oxaliplatin and fluorouracil chemotherapy. J Clin Oncol 19: 4298-4304, 2001.

20. Sobin LH and Wittekind C: TNM Classification of Malignant Tumours. 6th edition. New York, Wiley, 2002. 
21. Therasse P, Arbuck SG, Eisenhauer EA, Wanders J, Kaplan RS, Rubinstein L, Verweij J, Van Glabbeke M, van Oosterom AT, Christian MC and Gwyther SG: New guidelines to evaluate the response to treatment in solid tumors. J Natl Cancer Inst 92: 205-216, 2000.

22. Tischoff I, Hengge UR, Vieth M, Ell C, Stolte M, Weber A, Schmidt WE and Tannapfel A: Methylation of SOCS-3 and SOCS-1 in the carcinogenesis of Barrett's adenocarcinoma. Gut 56: 1047-1053, 2007.

23. Murai M, Toyota M, Suzuki H, et al: Aberrant methylation and silencing of the BNIP3 gene in colorectal and gastric cancer. Clin Cancer Res 11: 1021-1027, 2005.

24. Hocker M, Schlenger K, Hockel S and Vaupel P: Hypoxic cervical cancers with low apoptotic index are highly aggressive. Cancer Res 59: 4525-4528, 1999.

25. Hocker M and Vaupel P: Tumor hypoxia: definitions and current clinical, biologic, and molecular aspects. J Natl Cancer Inst 93 266-276, 2001.

26. Zagzag D, Zhong H, Scalziti JM, Laughner E, Simons JW and Semenza GL: Expression of hypoxia-inducible factor $1 \alpha$ in brain tumors: association with angiogenesis, invasion, and progression. Cancer 88: 2606-2618, 2000.

27. Blagosklonny MV: Antiangiogenic therapy and tumor progression. Cancer Cell 5: 13-17, 2004.

28. Manka D, Spicer Z and Millhorn DE: Bcl-2/Adenovirus E1B $19 \mathrm{kDa}$ interacting protein-3 knockdown enables growth of breast cancer metastases in the lung, liver, and bone. Cancer Res 65: 11689-11693, 2005.

29. Bruick RK: Expression of the gene encoding the proapoptotic Nip3 protein is induced by hypoxia. Proc Natl Acad Sci USA 97: 9082-9087, 2001.

30. Guo K, Searfoss G, Krolikowski D, Pagnoni M, Franks C, Clark K, Yu KT, Jaye M and Ivashchenko Y: Hypoxia induces the expression of the pro-apoptotic gene BNIP3. Cell Death Differ 8: 367-376, 2001.

31. Sowter HM, Ratcliffe PJ, Watson P, Greenberg AH and Harris AL: HIF-1-dependent regulation of hypoxic induction of the cell death factors BNIP3 and NIX in human tumors. Cancer Res 61: 6669-6673, 2001.

32. Bacon AL, Fox S, Turley H and Harris AL: Selective silencing of the hypoxia-inducible factor 1 target gene BNIP3 by histone deacetylation and methylation in colorectal cancer. Oncogene 26: 132-141, 2007.

33. De Angelis PM, Fjell B, Kravik KL, Haug T, Tunheim SH, Reichelt W, Beigi M, Clausen OP, Galteland E and Stokke T: Molecular characterizations of derivatives of HCT116 colorectal cancer cells that are resistant to the chemotherapeutic agent 5-fluorouracil. Int J Oncol 24: 1279-1288, 2004.
34. Kothari S, Cizeau J, McMillan-Ward E, Israels SJ, Bailes M, Ens K, Kirshenbaum LA and Gibson SB: BNIP3 plays a role in hypoxic cell death in human epithelial cells that is inhibited by growth factors EGF and IGF. Oncogene 22: 4734-4744, 2003.

35. Vande Velde C, Cizeau J, Dubik D, Alimonti J, Brown T, Israels S, Hakem R and Greenberg AH: BNIP3 and genetic control of necrosis-like cell death through the mitochondrial permeability transition pore. Mol Cell Biol 20: 5454-5468, 2000.

36. Okami J, Simeone DM and Logsdon CD: Silencing of the hypoxia-inducible cell death protein BNIP3 in pancreatic cancer. Cancer Res 64: 5338-5346, 2004.

37. Murai M, Toyota M, Satoh A, Suzuki H, Akino K, Mita H, Sasaki Y, Ishida T, Shen L, Garcia-Manero G, Issa JP, Hinoda Y, Tokino T and Imai K: Aberrant DNA methylation associated with silencing BNIP3 gene expression in haematopoietic tumors. Br J Cancer 92: 1165-1172, 2005.

38. Tan KB, Mattern MR, Eng WK, McCabe FL and Johnson RK: Nonproductive rearrangement of DNA Topoisomerase I and II genes: correlation with resistance to topoisomerase inhibitors. J Natl Cancer Inst 81: 1732-1735, 1989.

39. Bras-Gonçalves RA, Rosty C, Laurent-Puig P, Soulié P, Dutrillaux B and Poupon MF: Sensitivity to CPT-11 of xenografted human colorectal cancers as a function of microsatellite instability and p53 status. Br J Cancer 82: 913-923, 2000.

40. Schmaltz C, Harrigan PH, Wells A and Fisher DE: Regulation of proliferation-survival decisions during tumor cell hypoxia. Mol Cell Biol 18: 2845-2854, 1998.

41. Oizumi S, Isobe H, Ogura S, Ishida T, Yamazaki K, Nishimura M, Kawakami Y and Dosaka-Akita H: Topoisomerase inhibitorinduced apoptosis accompanied by down regulation of Bcl-2 in human lung cancer cells. Anticancer Res 22: 4029-4037, 2002.

42. Bani MR, Nicoletti MI, Alkharouf NW, Ghilardi C, Petersen D, Erba E, Sausville EA, Liu ET and Giavazzi R: Gene expression correlating with response to paclitaxel in ovarian carcinoma. Mol Cancer Ther 3: 111-121, 2004.

43. Erkan M, Kleeff J, Esposito I, Giese T, Ketterer K, Büchler MW, Giese NA and Friess H: Loss of BNIP3 expression is a late event in pancreatic cancer contributing to chemoresistance and worsened prognosis. Oncogene 24: 4421-4432, 2005.

44. Akada M, Crnogorac-Jurcevic T, Lattimore S, Mahon P, Lopes R, Sunamura M, Matsuno S and Lemoine NR: Intrinsic chemoresistance to gemcitabine is associated with decreased expression of BNIP3 in pancreatic cancer. Clin Cancer Res 11: 3094-3101, 2005

45. Chen G, Ray R, Dubik D, Shi L, Cizeau J, Bleackley RC, Saxena S, Gietz RD and Greenberg AH: The E1B 19K/bcl-2binding proteins Nip3 is a dimeric mitochondrial protein that activates apoptosis. J Exp Med 186: 1975-1983, 1997. 